\title{
Importance-Performance Analysis (IPA) for Technology-based Service: Sentiment Analysis Using User Review
}

\author{
Jieun Kim ${ }^{1}$ Yongtae Park ${ }^{1}$ Chulhyun Kim ${ }^{2}$ Moon-soo Kim ${ }^{3}$ \\ ${ }^{1}$ Department of Industrial Engineering, Seoul National University \\ ${ }^{2}$ Department of Technology \& Systems Management, Induk University \\ ${ }^{3}$ Department of Industrial \& Management Engineering, Hankuk University of Foreign \\ Studies
}

\begin{abstract}
In the era of technology-based service, a user review is one of significant source of diagnosing service qualities. The objective of this paper is to propose a user review based approach to developing the Importance-Performance Analysis (IPA) framework, which has been widely accepted as a tool for service quality improvement. Especially, this paper utilizes the sentiment analysis to measure the importance and performance from user reviews.
\end{abstract}

Keywords: Technology-based Service, Importance-Performance Analysis, Sentiment Analysis, User Review

\section{Introduction}

In the technology-based services which are mainly emerged in Information and Communication Technology (ICT) industry, users can actively participate in the service delivery, service evaluation, and even service development [1]. Particularly, since the users might easily comment on the services they experienced in the context of e-Service and m-Service, the user reviews are explosively emerged. Thus, it is crucial to monitor the opinions included in user reviews as a means of assessing the quality of services [2].
In the service quality literature, Importance-Performance Analysis (IPA) has been one of the significant models of service quality evaluation [3-4]. IPA can be plotted graphically using the importance and performance for each attribute, thus provide more practical guidance for strategic quality improvement [3]. Although it has been applied in various service sectors [4-6], there have been limited applications in the context of technologybased service. Moreover, a few studies of IPA for technology-based service still utilized survey rather than user review [5-6]. However, due to the usefulness of review data in terms of ampleness, reliability, influence, and accessibility [2], there is need for applying user reviews for IPA framework effectively.

In response, this paper suggests the user review based sentiment analysis approach to developing the IPA matrix. Especially, this paper constructs two types of keyword dictionaries from user reviews: attribute and sentiment dictionary. Also, in this paper, the frequency of attribute is defined as importance, whereas the sentiment score is defined as performance. Portfolio matrix identifies the current level of user satisfaction and compares it with competitors' quality.

\section{Literature Review}




\subsection{Importance-Performance Analy- sis}

The Importance-Performance Analysis (IPA) framework was introduced in marketing research in order to assist in understanding customer satisfaction as a function of both expectations concerning the significant attributes and judgments about their performance. IPA has been widely used in service industries such as tourism, education [4], finance [5], healthcare, etc. IPA is more superior to both SERVPERF and SERVQUAL because it has the importance attribution to the items and moreover, it can be plotted graphically using the importance and performance for each attribute [3]. This can be more powerful to provide practical guidance for strategic development.

The two dimensional IPA model is divided into four quadrants with performance on the $\mathrm{x}$-axis and importance on the y-axis. As a result of this, four quadrants are created, as depicted in Fig. 1. First, Concentrate Here represents key areas that need to be improved with top priority. Second, all attributes in Keep up the Good Work are the strength and pillar of the firms. Third, any of the attributes in Low Priority are not important and pose no threat to the firms. Last, Possible Overkill denotes attributes that are overly emphasized by the firms; therefore, firms should reallocate the resources used in this quadrant into first quadrant.

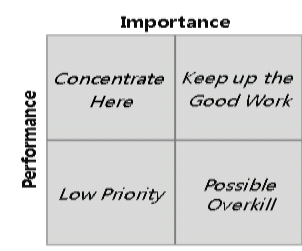

Fig. 1: IPA matrix

\subsection{Sentiment Analysis}

Sentiment analysis or opinion mining is the computational study of people's opinions, appraisals, and emotions toward entities, events and their attributes. Sentiment analysis provides firms with a means to estimate the extent of product acceptance and to determine strategies to improve product quality [8].

The essential issue in sentiment analysis is to identify how sentiments are expressed in texts and whether the expressions indicate positive or negative opinions toward the subject [9]. There exist two main approaches to extracting sentiment automatically [9]. The lexiconbased approach involves calculating orientation for a document from the semantic orientation of words or phrases in the document. The text classification approach involves building classifiers from labeled instances of texts or sentences, essentially a supervised classification task. We follow the first method, in which we use dictionaries of words annotated with the word's semantic polarity.

\section{Proposed approach}

This paper aims to develop IPA framework based sentiment analysis using user reviews. The overall research framework consists of four steps (Fig. 2).

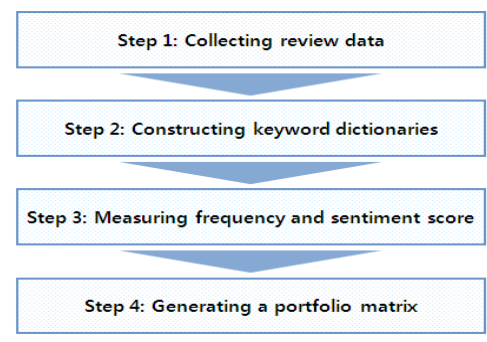

Fig. 2: Research process

First, review data are collected. Second, two types of keyword dictionaries are constructed: service attribute and sentimental words dictionary. Thirdly, the frequency and sentiment score are measured 
by sentiment analysis. In this paper, the frequency of attribute is defined as importance, whereas the sentiment score is defined as performance. Lastly, a portfolio matrix is generated by posing frequency and sentiment score in each axis. Portfolio matrix is utilized to identify the current level of customer satisfaction and compare it with competitors' quality.

\subsection{Collecting review data}

First of all, after selecting one service (which the focal firm provides), major competitors are selected. The reason why we include the competitor is that comparative evaluation of quality can be effective to provide ideas for improvement.

Then, user review data are collected from user communities for focal service and competitor's service. Since technology-based service firms have their own communities or users build up communities [1], there can be multiple communities for one service. For example, there are amount of mobile application review community websites such as "App shopper (www.appshopper.com)", and "App Safari (www.appsafari.com/)", etc. Thus, analyzer can select community sites which have quality user reviews.

\subsection{Constructing keyword dictionar- ies}

Since user review documents are crawled as text documents composed of HTML elements and real comments, data should be preprocessed as an analyzable DB.

After preprocessing, keywords are extracted from review texts to construct keyword dictionaries, including the dictionaries of service attributes and sentimental words. Service attribute dictionary involves the keywords regarding service features such as function, user interface, and price of service. Sentimental words dictionary includes the positive/negative sentiment words such as perfect, good, great, necessary, normal, terrible, etc. and modifiers expressing the degree of sentiment such as very, slightly, really, somewhat, most, etc.

\subsection{Measuring frequency and senti- ment score}

This paper investigates two measurements to identify the importance and performance of service attribute in user's perspective. First, the frequency of keywords from service attribute dictionary in the user review document is identified. This is defined as the importance in IPA, assuming that the more frequent the attribute keyword is mentioned in the user review, the more it is important regardless that it is positive or negative.

Second, the sentiment score is calculated. Sentences containing both sentimental words and service attribute words are extracted from review DB. For example, the sentence 'The search accuracy is very good' include service attribute words (i.e. 'search accuracy') as well as sentimental words ('very' and 'good'). The sentimental words are translated into sentimental score by defining scores for sentiment words (e.g. 'good' is 3 out of 5) and modifiers (e.g. 'very' adds 1). Since there are many opinions about one service attribute, total sentiment score is aggregated. This score is defined as the performance of IPA because it represents the level of user's satisfaction on the service attribute. The frequency and sentiment score are calculated for both focal service and competitive service.

\subsection{Generating a portfolio matrix}

Finally, the portfolio matrix is generated by posing the sentiment score (performance) on $\mathrm{x}$-axis and the frequency (importance) on y-axis, and by mapping the service attributes of focal firm (noted as filled circles) and competitor (signed as empty circles), as shown in the Fig 3. 
The portfolio matrix is interpreted in two perspectives. In terms of overall quality diagnosis, four quadrants are utilized as in the general IPA. In terms of competitive evaluation, the quality of service attributes is compared with competitors' service quality. For instance, the attribute $\mathrm{G}$ represents inferior sentiment score to competitor's attribute $G^{\prime}$ in spite of the similar frequency. Also, the attribute $I$ is the attribute that only the competitor has.

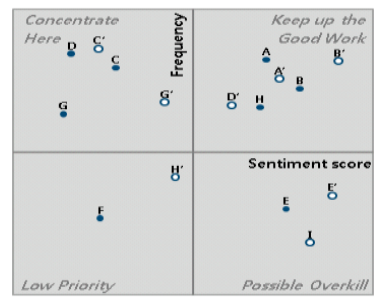

Fig. 3: Portfolio matrix

\section{Conclusion}

This paper proposed user review based sentiment analysis approach to IPA framework. This method can maximize the usefulness of user review and IPA in the service quality diagnosis in technology-based service. However, the future research should be conducted on fullscale case study and the detailed guidelines for extracting keywords and translating into sentiment scores.

\section{Acknowledgment}

This research was supported by Basic Science Research Program through the National Research Foundation of Korea (NRF) funded by the Ministry of Education, Science and Technology (2011-0007373).

\section{Reference}

[1] P. M. Di Gangi, and M. Wasko, "Steal my Idea! Organizational Adop- tion of User Innovations From a User Innovation Community: A Case Study of Dell IdeaStorm," Decision Support Systems, vol. 48, pp. 303-312, 2009.

[2] B. Bickart, and R.M. Schindler, "Internet Forums as Influential Sources of Consumer Information," Journal of Interactive Marketing, vol. 15, no. 2, pp. 31-40, 2001.

[3] N. Slack, "The ImportancePerformance Matrix as a Determinant of Improvement Priority," International Journal of Operations \& Production Management, vol. 14, no. 5, pp. 59-75, 1994.

[4] M. Joseph, and B. Joseph, "Service Quality in Education: A Student Perspective," Journal Quality Assurance in Education, vol. 5, no. 1, pp. 15-21, 1997.

[5] M. Joseph, D. Allbright, G. Stone, Y. Sekhon, and J. Tinson, "ImportancePerformance Analysis of UK and US Bank Customer Perceptions of Service Delivery Technologies," International Journal of Financial Services Management, vol. 1, pp. 66-88, 2005.

[6] M. S. Wong, N. Hideki, and P. George, "The Use of ImportancePerformance Analysis (IPA) in Evaluating Japan's E-government Services," Journal of Theoretical and Applied Electronic Commerce Research, vol. 6, no. 2, pp. 17-30, 2011.

[7] J. A. Martilla, and J. C. James, "Importance-Performance Analysis," Journal of Marketing, vol. 2, no. 1, pp. 77-79, 1977.

[8] R. Prabowo, and M. Thelwall, "Sentiment Analysis: A Combined Approach," Journal of Informetrics, vol. 3, no. 2, pp. 143-157, 2009.

[9] T. Nasukawa, and J. Yi, "Sentiment Analysis: Capturing Favorability Using Natural Language," Proc. Of the 2nd International Conference on Knowledge Capture, pp. 70-77, 2003. 